\title{
El rol del capital humano en el emprendimiento regional en Ecuador: un enfoque usando métodos espaciales
}

Rafael Alyarado lópez*, Cecibel Jiménez Soto**, Belén Sánchez Bustamante** y Pablo Ponce Ochoa*

\section{RESUMEN}

El emprendimiento es un mecanismo ampliamente utilizado para generar empleo y aumentar los niveles de producción. Sin embargo, no siempre surge de forma espontánea y más bien requiere del impulso de la política pública. En tal contexto, el objetivo de esta investigación es examinar el efecto del capital humano en la tasa de emprendimiento regional en Ecuador; utilizamos datos publicados por el Instituto Nacional de Estadísticas y Censos. Con el fin de capturar el contagio espacial que produce el nacimiento de nuevas empresas y la movilidad territorial del capital humano, estimamos un conjunto de modelos de rezago espacial (SAR), de error espacial (SEM), espacial de Durbin (SDM) y de rezago y error espacial (SARMA). Nuestros resultados destacan la importancia de la inclusión del rol del espacio en la comprensión de la interacción entre unidades territoriales y sugieren que el capital humano posee un efecto positivo en la tasa de emprendimiento regional con derrames espaciales. Los resultados son consistentes ante la inclusión de un conjunto de variables de control relacionadas con las dotaciones de las regiones. Una implicación de política pública derivada de nuestra investigación es que la planificación pública orientada a promover el nacimiento de nuevas empresas debe considerar el rol del contagio espacial del emprendimiento y la movilidad territorial del capital humano.

Palabras clave: Emprendimiento, capital humano, econometría espacial, Ecuador.

Clasificación JEL: L26. J24. C21.

\footnotetext{
* Profesores investigadores en la Carrera de Economía, Universidad Nacional de Loja, Ecuador Correo electrónico: jose.r.alvarado@unl.edu.ec y pablo.ponce@unl.edu.ec, respectivamente.

** Investigadoras en la Carrera de Economía, Universidad Nacional de Loja, Ecuador. Correo electrónico: cecibel.jimenez@unl.edu.ec y maria.b.sanchez@unl.edu.ec, respectivamente.
} 


\section{ABSTRACT}

The role of human capital in regional entrepreneurship in Ecuador: an approach using spatial methods

Entrepreneurship is a widely used mechanism to generate employment and increase production levels. However, it does not always arise spontaneously and rather requires the promotion of public policy. In this context, the objective of this article is to examine the effect of human capital on the rate of regional entrepreneurship in Ecuador. We use data published by the National Institute of Statistics and Census. In order to capture the spatial contagion produced by the emergence of new companies and the territorial mobility of human capital, we estimate a set of spatial lag (SAR), spatial error (SEM), Durbin spatial error (SDM) and lag and spatial error (SARMA) models. Our results show the importance of including the role of space in the understanding of the interactions between territorial units and suggest that human capital has a positive effect on the rate of regional entrepreneurship with spatial spillover. The results are consistent with the inclusion of a set of control variables related to the endowments of the regions. An implication of public policy derived from our research is that public planning aimed at promoting the emergence of new companies must consider the role of the spatial contagion of entrepreneurship and the territorial mobility of human capital.

Keywords: Entrepreneurship, Human Capital, Spatial Econometrics, Ecuador.

JEL classification: L26. J24. C21.

\section{INTRODUCCIÓN}

El emprendimiento es una de las formas más efectivas de dinamizar una economía y promover el progreso económico de las regiones o países. Sin embargo, en aquellos que se encuentran en desarrollo, un emprendedor enfrenta serios desafíos relacionados con la disponibilidad de capital humano calificado y con el financiamiento; además, debe efectuar varios trámites burocráticos antes de iniciar una actividad empresarial: para crear una empresa, se necesitan 56 días, trece pasos a seguir y alrededor de cinco salarios básicos mensuales (Banco Mundial, 2017); en las regiones con menor capital humano de Ecuador, la tasa de 
emprendimiento resulta extremadamente baja; pese a ello, la actividad emprendedora ecuatoriana mejoró durante los últimos años. En 2016, el índice de Actividad Emprendedora Temprana (TEA) fue de 31.8\%, 2.2 veces mayor al promedio de las economías de eficiencia; aunque esto representa una ligera baja en comparación con la de 2015, donde la TEA se ubicó en 33.6\%, Ecuador sigue siendo el país con la más alta en la región, y el segundo entre los 66 analizados en el "Global Report" (GEM, 2016). Resalta que el emprendimiento se concentra en los polos de desarrollo del país: Quito, Guayaquil y Cuenca, lo cual aumenta las disparidades territoriales, pues resulta limitado en las regiones más pobres del país.

Las trabas a las que se enfrentan los emprendedores refuerzan la necesidad de adquirir un conjunto de habilidades individuales y sociales para favorecer la generación de nuevas empresas. El conocimiento es el motor de partida de cualquier proceso de desarrollo, como lo formalizan los modelos de crecimiento endógeno (Lucas, 1988; Barro, 1992; Mankiw, Romer y Weil, 1992). La relación entre el emprendimiento y el capital humano constituye un tema abordado particularmente en los países de ingresos medio-alto y alto; la disponibilidad de datos confiables resulta un factor que contribuye a este crecimiento. Martin, McNally y Kay (2013), mediante una investigación meta-análisis, muestran que los aumentos del capital humano incrementan el nacimiento de empresas y el número de empresas de calidad; además, señalan que el capital humano y el emprendimiento se forman de manera conjunta. Dutta y Sobel (2018) ofrecen una posible explicación a este fenómeno: establecen que las personas con mayor capital humano tienen un acceso elevado al financiamiento y a otras formas de capital, como el social, adquirido por quienes cuentan con superiores niveles de educación; asimismo, Brush, Ali, Kelley y Greene (2017) sugieren que las capacidades y habilidades alcanzadas en la educación formal aumentan el emprendimiento de las mujeres.

En este escenario, la primera hipótesis verificada por nuestra investigación es que las regiones con mayor capital humano promedio presentan una tasa superior de emprendimiento. Jiménez y Alvarado (2017) y Jiménez y Alvarado (2018) utilizan técnicas de econometría espacial para demostrar la existencia de una fuerte interacción entre las unidades territoriales en Ecuador, en particular en relación con el capital humano, la producción y la especialización. En este sentido, la segunda 
hipótesis expresa que la actividad emprendedora genera contagio espacial y que la movilidad del capital humano influye positivamente en el emprendimiento de las regiones vecinas (Moreno-Serrano y Vayá, 2002); se basa en el enfoque de Faggian y Mccann (2009), quienes señalan que el capital resulta móvil en el territorio y, a medida que se torna más calificado, posee mayor interacción. Asumimos que la relación entre las dos variables es directa, pues cuando aumenta el capital humano, las personas se vuelven más competitivas, aprovechan de mejor forma los recursos existentes y con mayor eficiencia las oportunidades del lugar donde se localizan.

La primera hipótesis fue verificada en 84 países por Jiménez et al. (2015), quienes encuentran que la educación secundaria y superior impacta positivamente en la tasa de emprendimiento $\mathrm{y}$, a medida que aumenta el capital humano, también lo hace el emprendimiento formal y se reduce el informal. La segunda hipótesis se basa en el hecho de que el capital humano es móvil en el espacio (Faggian y McCann, 2009; Jiménez y Alvarado, 2017; Jiménez y Alvarado, 2018): resulta evidente que el emprendimiento de una región genera contagio espacial en las más próximas (Piacentino, et al., 2017) debido a que las empresas demandan insumos y venden su producción en el lugar donde se localizan y en los sitios vecinos. Finalmente, el comportamiento de los datos de la economía ecuatoriana sustenta estas hipótesis.

Los avances recientes en econometría espacial permiten capturar la interacción espacial entre unidades territoriales. En todos los modelos estimados, la variable dependiente es la tasa de emprendimiento y la independiente, el capital humano; los datos corresponden a la tasa de emprendimiento en un periodo de cinco (2006-2010) y de diez años (2001-2010). Los resultados encontrados sugieren que la omisión de la dependencia espacial genera inconsistencias, debido a que el test de I-Moran señala la existencia de dependencia espacial; cuando se utiliza un modelo de rezago espacial (SAR), encontramos que el capital humano produce un efecto positivo y significativo en la tasa de emprendimiento regional, lo que coincide con las conclusiones obtenidas por Wu y Huarng (2015) y Devece, Ortiz y Armengot (2016). Cuando estimamos un modelo de error espacial (SEM), encontramos que la tasa de emprendimiento de una región depende del error de sus cantones vecinos; esto implica que aquella se explica por variables no incluidas en el modelo; por lo tanto, se incorporan covariantes adicionales que 
reflejan las dotaciones de las regiones; los resultados del modelo Durbin (SDM) confirman las conclusiones previas. El modelo de error y de rezago espacial (SARMA) sugiere que la tasa de emprendimiento de una región depende del capital humano promedio de los cantones vecinos; ello concuerda con las conclusiones conseguidas por Onkelinx, Manolova y Edelma (2016). Los resultados encontrados resaltan la importancia del capital humano como un factor clave en la generación de la actividad emprendedora regional.

Nuestra investigación aporta al debate sobre la relación entre el capital humano y la actividad emprendedora con evidencia empírica a partir de una amplia base de datos censales para un país en desarrollo, cuyas características estructurales y limitaciones enfrentadas por los emprendedores difieren de la realidad de las naciones desarrolladas. Además, la literatura se ha enfocado en medir el efecto del capital humano en la tasa de emprendimiento regional utilizando regresiones tradicionales (Martin, McNally y Kay, 2013; Batjargal, 2007) que omiten la interacción territorial, por lo que los parámetros podrían ser inconsistentes y sesgados. En general, nuestros resultados indican que la tasa de emprendimiento de una región depende de ésta y del capital humano promedio de las zonas vecinas. Las implicaciones de políticas públicas que sustentan estas deducciones residen en que, para acelerar el desarrollo de los lugares pobres mediante el nacimiento de nuevas empresas, se debe aumentar la inversión en capital humano en los sitios periféricos. Los beneficios ocurren de manera directa en las mismas regiones y en las aledañas debido a los efectos del contagio espacial.

El resto de esta investigación tiene la siguiente estructura: revisión de la literatura previa; descripción de las fuentes estadísticas y planteamiento de la estrategia econométrica; discusión de los resultados encontrados a partir de la teoría y la evidencia empírica previa; finalmente, las conclusiones e implicaciones de política.

\section{REVISIÓN DE LA LITERATURA PREVIA}

Diversos factores explican el surgimiento de nuevas empresas; por una parte, se argumenta que el emprendimiento aparece por la necesidad de los individuos de alcanzar una independencia laboral o, en algunos casos, de encontrar un empleo (Formichella, 2004); otros enfoques proponen que el emprendimiento se explica sólidamente por factores 
motivacionales (Marulanda-Valencia, Montoya-Restrepo y VélezRestrepo, 2014), como la necesidad de éxito (Mejía-Cubillos, 2013; Díaz-García y Jiménez-Moreno, 2010; Huggins, Prokop y Thompson, 2017). La lógica de la teoría que relaciona el capital humano con el emprendimiento es que la educación, además de proveer conocimientos e información, cambia atributos y actitudes, lo cual puede forjar cualidades emprendedoras: si los individuos se encuentran capacitados tendrán mayor facilidad para emprender nuevos negocios que ayuden a generar nuevos empleos, a aumentar los ingresos y, por lo tanto, contribuirán a la dinamización económica regional y nacional.

La relación entre el capital humano y la tasa de emprendimiento se ha incrementado en los últimos años; la evidencia empírica que verifica la relación entre las dos variables se divide en tres grupos; el primero involucra el efecto positivo del capital humano, donde destacan los trabajos realizados por Ahsan y Haque (2017) y Mestieri, Schauer y Townsend (2017), quienes indican que un individuo, para desarrollarse en el ámbito empresarial, debe contar con educación, capacidades y habilidades que le permitan desempeñarse de forma adecuada en el mercado laboral, con la subsecuente generación de ingresos que mejoren la calidad de vida familiar y de la sociedad en general. En este sentido, existe una amplia literatura reciente que resalta tal vinculación y su impacto regional (Bobonis y Morrow, 2014; Attanasio, et al., 2017; Weldeegzie, 2017).

El segundo grupo analia la trascendencia del emprendimiento como mecanismo de progreso; por ejemplo, Lucas y Fuller (2017) y Lim, Oh y De Clercq (2016), luego de estimar sus modelos, obtuvieron que el capital humano cobra gran importancia para la existencia del emprendimiento: cuando una persona cuenta con los suficientes conocimientos podrá incluirse en cualquier actividad económica en beneficio del crecimiento y desarrollo económico. Silveira-Pérez, Cabeza-Pullés y Fernández-Pérez (2016) y Yolac (2015) coinciden con las investigaciones realizadas anteriormente, pero sugieren que el apoyo del Estado es necesario para la creación de micro empresas por parte de pequeños grupos de emprendedores. Por su parte, Wu y Huang (2015), Devece, Ortiz y Armengot (2016), y Urbano y Aparicio (2016) aportan que el espíritu empresarial impacta en el desarrollo de un país porque crea actividades económicas tendientes a generar nuevos puestos de trabajo 
$\mathrm{y}$, por lo tanto, a aumentar la riqueza del territorio y disminuir del desempleo.

En el tercer grupo, se ubican las investigaciones realizadas por Martin, McNally y Kay (2013), Batjargal (2007) y Estrin, Mikiewicz y Stephan (2016), quienes analizan cómo influye el capital humano en el emprendimiento, con un efecto positivo revelador como resultado: a medida que aumentan las capacidades y habilidades de los individuos, éstos tendrán mayores posibilidades de crear pequeñas y medianas empresas. Lin y Yang (2017), Yang y An (2002) y Backes-Gellner y Moog (2013) coinciden con los autores anteriores, si bien destacan que la mayoría de los emprendimientos son iniciados por personas que han obtenido una educación superior, pues al contar con conocimientos nuevos han sido capaces de crear empresas propias sin dificultad, generando beneficios y, a su vez, puestos de trabajo para la sociedad. Finalmente, Salazar, Hidalgo y Manríquez (2017) y Onkelinx, Manolova y Edelma (2016) refuerzan la idea de que el capital humano juega un rol central para iniciar un emprendimiento.

Investigaciones realizadas recientemente han profundizado la explicación del nexo causal entre el capital humano y el emprendimiento mediante una argumentación teórica y empírica. A pesar de la falta de unanimidad en los resultados de la evidencia empírica, en el ámbito teórico existe un consenso sobre la importancia de la educación en la mejora de las habilidades y actitudes individuales que conducen a tal actividad. Por ejemplo, Martin, McNally y Kay (2013), luego de una amplia revisión de las investigaciones que relacionan ambas variables, coligen que la mayor parte llega a dos conclusiones: por un lado, los aumentos en el capital humano se traducen en un incremento de la tasa de emprendimiento, de modo que los países, regiones e individuos con mayor capital humano presentan tasas superiores; por otro, las elevaciones del capital humano contribuyen a mejorar la calidad de los nuevos emprendimientos. El enfoque de emprendimiento por necesidades u oportunidades es compatible con ambas posturas, pues el proceso de creación se refuerza gracias a las dotaciones de capital humano, en particular en los proyectos relacionados con nuevas tecnologías, servicios y, en alguna medida, los generados por los inmigrantes (Lin y Yang, 2017).

El rol de las instituciones formales resulta clave en el establecimiento de nuevas empresas: las personas que pueden aprovechar de 
mejor forma su apoyo son aquellas que están en condiciones de apropiarse de la información y de recibir el respaldo del Estado o de organizaciones sin fines de lucro; Dutta y Sobel (2018) ofrecen otra explicación que sustenta esta relación positiva: las personas con capital humano superior tienen mayor acceso al sistema financiero y crediticio, además de otras formas de capital, como el social. Alvarado, et al. (2017) muestran que al acceso al financiamiento determina, en parte, la cantidad de nacimientos de empresas; dado que los emprendedores requieren de un capital para iniciarlas y mantenerlas en funcionamiento, la relación que verifican Dutta y Sobel (2018) resulta coherente con la hipótesis que plantea esta investigación: se ha demostrado que mediante la educación se desarrollan y refuerzan las habilidades y capacidades naturales de las personas, incluyendo las necesarias para recurrir a las instituciones que apoyan el emprendimiento y concluir los procesos destinados a tales fines; en este sentido, Brush, et al. (2017) muestran que la educación formal lleva a que las personas adquieran más capacidades y habilidades y esto, a su vez, aumenta el emprendimiento, en particular en el caso de las mujeres.

La segunda relación planteada en la presente investigación, a saber, que el emprendimiento y el capital humano implican un contagio espacial, posee un amplio respaldo empírico; en parte, porque el espíritu empresarial reflejado en la formación de nuevas empresas y el capital humano son ampliamente reconocidos como motores del desarrollo económico regional (Qian y Zhao, 2018; Huachizaca y Alvarado, 2018; Cueva y Alvarado, 2017). Sin embargo, varias investigaciones previas omiten la interacción espacial entre unidades territoriales, aunque sea evidente que en la práctica no actúan como islas, sino que existen flujos de conmutación, de mercaderías, de personas, de capitales, entre otros (Jiménez y Alvarado, 2017; Jiménez y Alvarado, 2018). Por una parte, la evidencia empírica sugiere que el capital humano no permanece estático en el espacio: a medida que se vuelve más calificado, aumenta su movilidad en el territorio (Faggian y McCann, 2009); por otra, dado que las economías tienden a incrementar la participación del sector terciario, la oferta y demanda de servicios asociados con el capital humano presentarían una fuerte interrelación con el lugar. Plummer y Acs (2014) encuentran una articulación positiva entre los nuevos conocimientos y la actividad emprendedora: demuestran la existencia de contagio espacial de la actividad emprendedora. 
Läpple, et al. (2016) consideran al capital humano como el factor que genera el contagio espacial del emprendimiento; a conclusión similar llega Cantú (2017), quien señala que los emprendimientos generan un efecto spillover positivo mediante las interrelaciones con los proveedores de insumos y los demandantes de sus productos, en tanto no necesariamente se localizan en el lugar del emprendimiento. El rol de las instituciones educativas como proveedoras del capital humano trasciende de modo particular para la actividad emprendedora de una región; por ejemplo, Audretsch y Lehmann (2005) argumentan que el número de empresas ubicadas cerca de una universidad está influido positivamente por la capacidad de conocimiento de esta región y la producción de conocimiento de una universidad. La revisión de la literatura sugiere que el capital humano y el nacimiento de nuevas empresas poseen una relación positiva y que el emprendimiento genera un contagio positivo en las regiones aledañas; ambas hipótesis son verificadas para el caso de Ecuador con datos censales como base.

\section{FUENTES ESTADÍSTICAS Y ESTRATEGIA ECONOMÉTRICA}

\subsection{Datos}

Esta investigación utiliza información secundaria tomada de las bases de datos del Instituto Nacional de Estadísticas y Censos (INEC) y del Sistema Integrado de Indicadores Sociales de Ecuador (SIISE, 2008). La variable dependiente es la tasa de emprendimiento medida de dos formas: la primera toma en cuenta las empresas que nacieron en el periodo 2006-2010 y la segunda incluye al grupo surgido entre 2001 y 2010; considerar ambas asegura la ampliación temporal y, por consiguiente, ofrece mayor consistencia de resultados; todos los datos de las empresas corresponden a información recogida en el Censo Nacional Económico de 2010.

La variable independiente (capital humano) también implica información censal; se construye a partir de dos variables: nivel de instrucción y número de años aprobados por los individuos que residen en cada cantón del país. En el proceso de construcción de la variable escolaridad como medida del capital humano, el nivel de instrucción contiene diez categorías que denotan los distintos grados de educación en forma secuencial. La variable año aprobado representa el número 
de años que ha cursado la población dentro de cada nivel de instrucción. En Ecuador, el capital humano tiene un efecto significativo en la producción agregada (Mora, et al., 2017), sin embargo, es necesario estimar la importancia del capital humano en el comportamiento de una economía a partir de la interacción espacial. Finalmente, se adicionaron diversas variables de control que reflejan las dotaciones de las regiones, tales como su capacidad exportadora, el ingreso per cápita, el gasto en capacitación y formación y la tasa de urbanización.

La Tabla 1 resume la descripción de las variables.

Tabla 1

DEFINICIÓN Y DESCRIPCIÓN DE LAS VARIABLES

\begin{tabular}{ccc}
\hline Variables & Descripción & Unidad de Medida \\
Tasa de emprendimiento & $\begin{array}{c}\text { Es aquella actitud y aptitud que toma un } \\
\text { individuo para iniciar un nuevo proyecto a } \\
\text { través de ideas y oportunidades. }\end{array}$ & Tasa de participación regional
\end{tabular}

$\begin{array}{ccc}\text { Capital humano } & \begin{array}{l}\text { Es una medida del valor económico de las } \\ \text { habilidades profesionales de una persona }\end{array} & \text { Años de escolaridad promedio } \\ \text { por región }\end{array}$

Se define como el envío de un producto
o servicio a un país extranjero con fines Tasa de participación regional
comerciales.

\begin{tabular}{ccc} 
Ingreso per cápita & $\begin{array}{c}\text { Es un cálculo que se realiza para } \\
\text { determinar el ingreso que recibe en } \\
\text { promedio cada uno de los habitantes de } \\
\text { un país. }\end{array}$ & $\begin{array}{c}\text { Logaritmo del ingreso per- } \\
\text { cápita, expresada en unidades } \\
\text { monetarias constantes }\end{array}$ \\
$\begin{array}{c}\text { Gasto en capacidad y } \\
\text { formación }\end{array}$ & $\begin{array}{c}\text { Es el proceso a través del cual se obtienen } \\
\text { fortalezas y capacidades para establecer y y } \\
\text { lograr sus propios objetivos. }\end{array}$ & Tasa de participación regional \\
Población urbana & $\begin{array}{c}\text { Se considera que una población urbana se } \\
\text { caracteriza por estar habitada en forma } \\
\text { permanente por más de 2 mil habitantes. }\end{array}$ & Tasa de población urbana \\
\hline
\end{tabular}

La capacidad exportadora de cada región muestra el potencial para generar ingresos provenientes del exterior; se espera que su vinculación con la tasa de emprendimiento resulte positiva. Asimismo, se asume la presencia de una relación positiva entre el emprendimiento y el ingreso per cápita debido a que, cuando éste es superior, se presentan 
mayores oportunidades para emprender. Para evitar la endogeneidad con la variable dependiente se emplean las tasas de participación y el logaritmo, respectivamente. Un resultado similar se da con la tasa de urbanización y el gasto en capacitación y formación; la primera puede capturar las ventajas para las nuevas empresas de la concentración de la población en los centros urbanos y, la segunda, reflejar las capacidades $\mathrm{y}$ habilidades adicionales al capital humano que posee la fuerza laboral de cada región.

La Figura 1 expresa la relación entre el emprendimiento por cinco años de las empresas y el capital humano en la región; a medida que hay mayor tasa de emprendimiento, el color se torna más intenso y viceversa. El capital humano se representa con los círculos: los más pequeños indican su escasez, de modo que el tamaño crece de acuerdo con el incremento de su presencia.

La Tabla 2 muestra el resumen de las variables utilizadas; cabe resaltar que el modelo cuenta con 218 cantones (fueron omitidos tres por la limitación de datos confiables). Además, se indican los estadísticos descriptivos de las variables (como la media, la desviación estándar, el valor máximo y mínimo) de aquellas empresas con cinco años en el mercado.

Tabla 2

ESTADÍSTICOS DESCRIPTIVOS DE LAS VARIABLES

\begin{tabular}{cccccc}
\hline Variable & Observaciones & Media & D.S. & Mínimo & Máximo \\
\hline Log(emprendimiento5) & 218 & 5.7 & 1.4 & 2.3 & 11.29 \\
\hline Log(emprendimiento10) & 218 & 6.13 & 1.4 & 2.3 & 11.29 \\
\hline Log(capital humano) & 218 & 4.18 & 1.75 & 0.69 & 10.8 \\
\hline Tasa(capacitación y formación) & 218 & 2.85 & 2.14 & 0 & 18.18 \\
\hline Log (exportación) & 218 & 0.73 & 1.27 & 0 & 6.5 \\
\hline Población urbana & 218 & 0.38 & 0.22 & 0.05 & 1 \\
\hline Log(Ingresos per-cápita) & 218 & 7.08 & 1.03 & 4.74 & 10.28 \\
\hline
\end{tabular}

Se observa gráficamente que en los cantones donde el emprendimiento es mayor, el capital humano también resulta alto y viceversa; sin embargo, en algunos casos, éste es bajo aun con emprendimiento elevado. Tal disonancia puede deberse a que existen regiones donde los emprendedores no necesariamente tienen mayor cualificación formal: 
han logrado concretar sus proyectos a pesar de las limitaciones del capital humano.

Figura 1

EMPRENDIMIENTO Y CAPITAL HUMANO A NIVEL REGIONAL ECUADOR

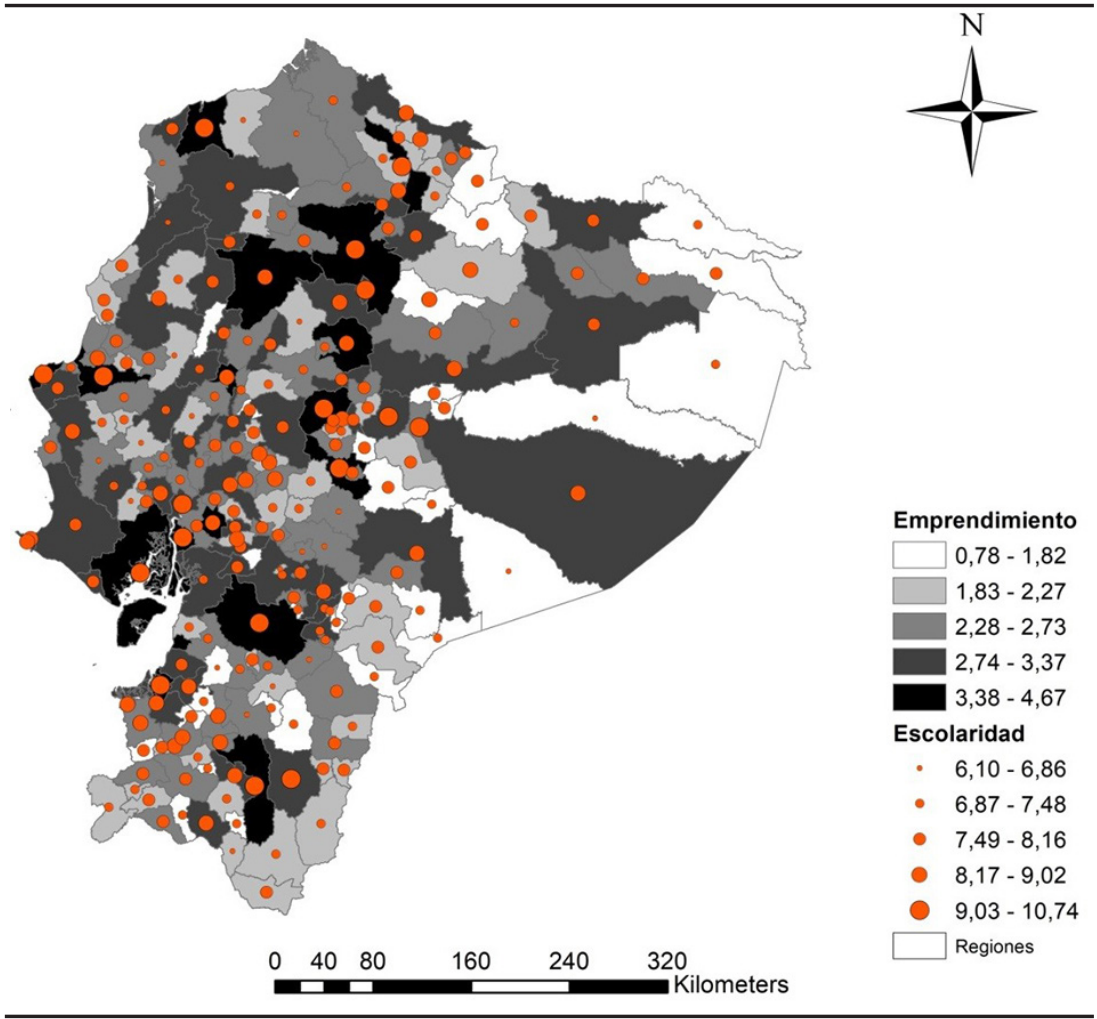

Fuente: Elaboración propia con datos del INEC, 2010.

La vinculación entre el emprendimiento a diez años y el capital humano es idéntica a la previa; las estimaciones econométricas presentadas en la siguiente sección confirman la relación inicial mostrada en la Figura 1. Siguiendo a Aroca (2000), la Tabla 3 reporta los resultados del test de dependencia espacial para verificar formalmente la existencia de la necesidad de incluir los modelos que capturan la dependencia espacial de las variables capital humano (a 5 y 10 años) y tasa de emprendimiento. 
Tabla 3

RESULTADOS DEL TEST DE DEPENDENCIA ESPACIAL

\begin{tabular}{|c|c|c|c|c|c|c|c|c|c|}
\hline \multirow[b]{2}{*}{ TEST } & \multicolumn{3}{|c|}{ Emprendimiento (5 años) } & \multicolumn{3}{|c|}{ Emprendimiento (10 años) } & \multicolumn{3}{|c|}{ Capital humano } \\
\hline & $\mathrm{Mi} / \mathrm{df}$ & Value & Prob. & $\mathrm{Mi} / \mathrm{df}$ & Value & Prob. & $\mathrm{Mi} / \mathrm{df}$ & Value & Prob. \\
\hline $\begin{array}{l}\text { Moran's I } \\
\text { (error) }\end{array}$ & 0.419 & 7.87 & 0.000 & 0.430 & 10.158 & 0.000 & 0.512 & 12.068 & 0.000 \\
\hline $\begin{array}{c}\text { Lagrange } \\
\text { Multiplier (lag) }\end{array}$ & 1 & 23.15 & 0.000 & 1 & 48.184 & 0.000 & 1 & 27.153 & 0.000 \\
\hline Robust LM (lag) & 1 & 0.193 & 0.030 & 1 & 21.182 & 0.031 & & & \\
\hline \multicolumn{10}{|l|}{ Lagrange } \\
\hline $\begin{array}{l}\text { Multiplier } \\
\text { (error) }\end{array}$ & 1 & 138.809 & 0.000 & 1 & 39.130 & 0.000 & 1 & 0.136 & 0.713 \\
\hline $\begin{array}{l}\text { Robust LM } \\
\text { (error) }\end{array}$ & 1 & 111.792 & 0.000 & 1 & 5.108 & 0.024 & & & \\
\hline \multicolumn{10}{|l|}{ Lagrange } \\
\hline $\begin{array}{l}\text { Multiplier } \\
\text { (SARMA) }\end{array}$ & 2 & 138.945 & 0.000 & 2 & 55.454 & 0.000 & & & \\
\hline
\end{tabular}

Fuente: Elaboración propia con datos del INEC, 2010.

\subsection{Metodología}

Los avances de la econometría espacial a partir de los trabajos realizados por Anselin (1988) permiten incorporar el rol de la interacción entre las unidades territoriales en la comprensión del comportamiento de las variables sociales y económicas. En años recientes surgió una literatura empírica tendiente a apuntar que la omisión de la dependencia espacial puede propiciar estimadores inconsistentes. Las unidades territoriales no funcionan como islas, más bien se implican en grandes flujos de comercio, de personas y de capitales; omitirlos lleva no solo a obtener parámetros sesgados, sino a recomendar políticas económicas erróneas.

En tal contexto, el objetivo de esta investigación se divide en dos partes: evaluar el efecto del rol del capital humano en la tasa de emprendimiento regional y plantear un modelo que capture su contagio espacial hacia las regiones vecinas. Varios trabajos recientes han verificado que considerar la dependencia espacial mejora notablemente los estimadores y contribuye a entender integralmente los procesos de desarrollo regional (Jiménez y Alvarado, 2017; Jiménez y Alvarado, 2018). La siguiente ecuación formaliza la primera relación: 


$$
E m p_{j}=\beta_{0}+\beta_{1} C h_{j}+\varepsilon_{j}
$$

En la ecuación (1), Emp $\mathrm{j}$ es la tasa de emprendimiento; $\mathrm{Ch}_{\mathrm{j}}$, el capital humano promedio cantonal; finalmente, $\varepsilon_{\mathrm{j}}$, el término de error estocástico. La variable dependiente, la tasa de emprendimiento, ha sido considerada en dos grupos a partir del lapso de constitución de las empresas: de cero a cinco años (2006-2010) y de cero a diez años (2001-2010). En la práctica, existen otros factores distintos al capital humano que inciden en el nacimiento de nuevas empresas; con el fin de capturar esos efectos y verificar si los resultados son estables, fueron incluidas cuatro variables de control; con ellas, el modelo (1) se convierte en uno más amplio, como lo formaliza la siguiente ecuación:

$$
E m p_{j}=\beta_{0}+\beta_{1} C h_{j}+\beta_{2} C f_{j}+\beta_{3} X_{j}+\beta_{4} P u_{j}+\beta_{5} \log I p_{j}+\varepsilon_{j}
$$

En la Ecuación (2), Emp ${ }_{\mathrm{j}}$ es la tasa de emprendimiento; $\mathrm{Ch}_{\mathrm{j}}$, el capital humano; $\mathrm{Cf}_{\mathrm{j}}$, la tasa de gasto en capacitación y formación; $\mathrm{X}_{\mathrm{j}}$, las exportaciones; $\mathrm{Pu}_{\mathrm{j}}$, la tasa de urbanización; $\mathrm{Ip}_{\mathrm{j}}$, el ingreso per cápita; finalmente, $\varepsilon_{\mathrm{j}}$, el término de error estocástico que se asume sigue una distribución con media cero y varianza constante $\left(0, \sigma^{2}\right)$.

Sobre la segunda relación referida supra, para formalizar la interacción espacial entre las regiones con respecto a las variables incluidas en la presente investigación, verificamos formalmente la dependencia espacial a través del índice I-Moran. Lee (2001) señala que el I-Moran global es un índice de covariación entre regiones y fluctúa entre -1 y 1; cuando tiende a 0 implica que no hay autocorrelación espacial, mientras que si lo hace a -1 o a 1 está presente la autocorrelación espacial. La fórmula de cálculo del I-Moran es la siguiente:

$$
I=\frac{N \sum_{j} \sum_{j} w_{i j}\left(x_{i}-\bar{x}\right)\left(x_{j}-\bar{x}\right)}{\sum_{j} \sum_{j} w_{i j}\left(x_{i}-\bar{x}\right)}
$$

En la ecuación (3), $\mathrm{N}$ es el número de regiones; $\mathrm{x}_{\mathrm{i}}$, el valor de la variable en la región $\mathrm{i} ; \mathrm{x}_{\mathrm{j}}$, el valor de la variable en promedio en el resto de regiones $\mathrm{j} ; \overline{\mathrm{x}}$, la media de la variable analizada; $\mathrm{w}_{\mathrm{ij}}$, un peso que depende de localización de las observaciones. En la investigación medimos la dependencia espacial del capital humano y tasa de emprendimiento: a partir de Anselin (1988), se considera que $\mathrm{w}_{\mathrm{ij}}$ captura la interacción 
espacial entre cada par de unidades geográficas; utilizamos el criterio de contigüidad de las cinco regiones más próximas. La dependencia espacial implica que los valores observados en una región dependen de los de los sitios aledaños.

De acuerdo con Jiménez y Alvarado (2017), el modelo SAR permite determinar cómo la tasa de emprendimiento de una región es afectada por la de las zonas vecinas. Asimismo, el modelo SEM muestra cómo la tasa de emprendimiento de una región resulta influida por variables omitidas en los sitios cercanos. Finalmente, el modelo SARMA combina SAR y SEM.

La elección del modelo por estimar fue realizada en función de las pruebas robustas de dependencia espacial; su base radica en que las relaciones entre regiones influyen para que los valores que toma una dependan de los tomados por las vecinas. De acuerdo con Anselin (1988) y Chasco (2003), un modelo SAR puede ser planteado en formato matricial de la siguiente forma:

$$
\log (y)=\alpha_{0}+\rho W y+X \beta+\varepsilon
$$

En la ecuación (4), el término $\mathrm{W}_{\mathrm{y}}$ es un vector de $\mathrm{N}$ por 1 de rezagos espaciales para la variable dependiente; $\rho$, el coeficiente autorregresivo espacial; y $\varepsilon$, un vector de $\mathrm{N}$ por 1 del error normalmente distribuido, con media 0 y varianza $\sigma^{2}$. El parámetro escalar $\rho$ toma un valor de cero cuando no hay dependencia espacial; el término $\rho \mathrm{W} y$ es una combinación lineal de los valores de la variable y elaborada para que capture el contagio espacial de la variable formalizada. Además, X es una matriz de covariantes planteada en las fuentes estadísticas; incluye la tasa de urbanización, las exportaciones, el gasto en capacitación y formación y el ingreso per cápita. Un modelo SEM tiene la siguiente forma:

$$
\log (y)=\alpha_{0}+X \beta+\lambda W u+\theta
$$

En la ecuación (5), el parámetro $\lambda$ captura el efecto de las variables omitidas en la variable dependiente. Finalmente, el modelo SARMA combina las ecuaciones (4) y (5) como sigue:

$$
\log (y)=\alpha_{0}+\rho W y+\lambda W u+X \beta+\theta
$$


En suma, la inclusión de los modelos de dependencia espacial está justificada porque los valores que toman las variables en la región están afectados por lo que ocurre en los territorios vecinos (Baronio, Vianco y Rabanal, 2012). Así, el modelo espacial de Durbin (SDM) puede ser obtenido a partir de las ecuaciones previas. Los resultados recogidos de las estimaciones de los modelos antes planteados se reportan y discuten en la siguiente sección.

\section{Discusión de resultados}

La Figura 3 muestra el diagrama de dispersión de Moran para la tasa de emprendimiento de cinco y diez años; además, expresa el Moran del capital humano promedio regional. El resultado obtenido para la tasa de emprendimiento, tanto para cinco como diez años, es positivo y estadísticamente significativo ya que el $p$-value $=0.001$ y 0.03 para el capital humano. En la práctica, las tasas de emprendimiento y del capital humano de un cantón influyen positivamente en las de las zonas aledañas. Esto implica que existe autocorrelación espacial positiva en la tasa de emprendimiento y en el capital humano promedio regional, por lo tanto, se debe incorporar la interacción espacial para corregir el sesgo producido por la omisión de la dependencia espacial de los modelos MCO.

Figura 2

\section{DIAGRAMA DE MORAN DE LA TASA DE EMPRENDIMIENTO Y DEL CAPITAL HUMANO}
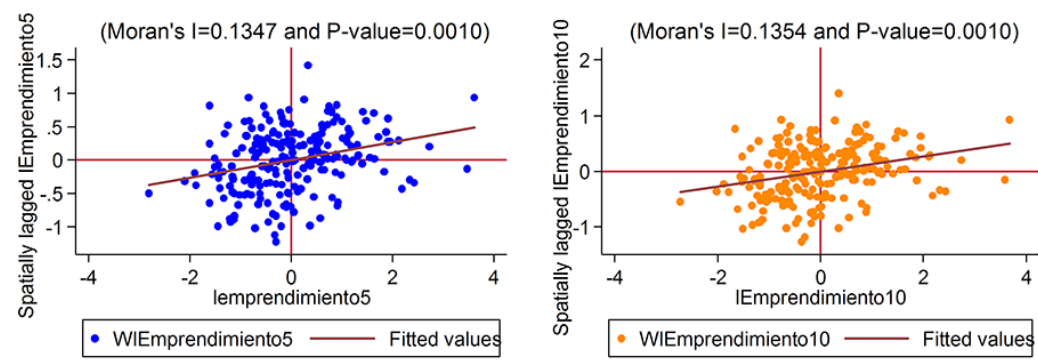


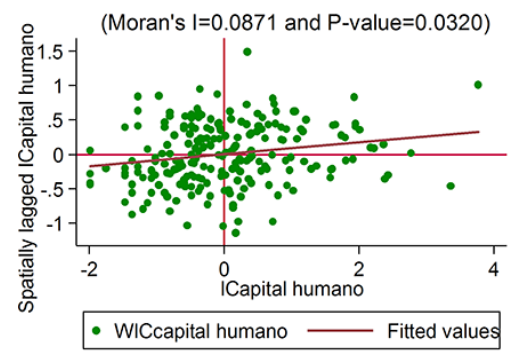

Fuente: Elaboración propia con datos del INEC, 2010.

La Tabla 4 indica, mediante los cuatro métodos espaciales, los resultados obtenidos en el modelo estimado cuyo tiempo de emprendimiento en el mercado es de cinco y diez años. Para los modelos estimados, en las regiones que cuentan con mayores años de escolaridad el emprendimiento en ambos lapsos muestra una relación positiva: cuando el capital humano varía en $1 \%$, el emprendimiento lo hace entre $0.56 \%$ y $0.57 \%$. De igual manera, se mantiene la relación positiva entre las exportaciones y el emprendimiento, puesto que una elevada apertura comercial motiva a aumentar las actividades en este sentido: una modificación de $1 \%$ en las exportaciones provoca una variación entre $0.10 \%$ y $0.13 \%$. A su vez, cuando el ingreso per cápita resulta más alto, el emprendimiento aumenta, en tanto los individuos disponen de mayores recursos para emprender: el incremento del 1\% del ingreso per cápita ocasiona un incremento de entre $0.15 \%$ y $0.16 \%$. Cuando las personas residen en área urbana el emprendimiento asciende debido a la calidad de vida y a las facilidades que otorga habitar en una ciudad: un cambio en el $1 \%$ de la variable urbanización provoca que el emprendimiento varíe entre $0.66 \%$ y $0.79 \%$. Finalmente, las regiones que denotan bajos niveles de capacitación son las que evidencian mayor emprendimiento, lo cual puede explicarse porque en algunos escenarios la experiencia es un factor determinante a la hora de establecer nuevos negocios; por ello, el aumento de $1 \%$ en capacitación y formación provoca una disminución del emprendimiento entre $0.074 \%$ y $0.078 \%$. La literatura empírica sugiere que el capital humano en Ecuador no se distribuye aleatoriamente en el territorio; en particular, el calificado posee una alta concentración espacial (Cueva y Alvarado, 2017). 
Tabla 4

RESULTADOS DE LOS MODELOS ESPACIALES

\begin{tabular}{|c|c|c|c|c|c|c|c|c|}
\hline & SAR5 & SEM5 & SDM5 & SARMA5 & SAR10 & SEM10 & SDM10 & SARMA10 \\
\hline Capital humano & $\begin{array}{l}0,72 * * * \\
(-35,21)\end{array}$ & $\begin{array}{l}0,73 * * * \\
(-34,67)\end{array}$ & $\begin{array}{l}0,72 * * * \\
(-35,29)\end{array}$ & $\begin{array}{l}0,72 * * * \\
(-35,42)\end{array}$ & $\begin{array}{c}0,73 * * * \\
(-36,1)\end{array}$ & $\begin{array}{l}0,73 * * * \\
(-35,76)\end{array}$ & $\begin{array}{l}0,73 * * * \\
(-36,36)\end{array}$ & $\begin{array}{l}0,73 * * * \\
(-36,55)\end{array}$ \\
\hline Constant & $\begin{array}{c}1,71 * * * \\
(-5,87)\end{array}$ & $\begin{array}{l}2,67 * * * \\
(-26,95)\end{array}$ & $\begin{array}{c}1,64 * * * \\
(-5,26)\end{array}$ & $\begin{array}{c}1,80 * * * \\
(-5,49)\end{array}$ & $\begin{array}{c}2,01 * * * \\
(-6,58)\end{array}$ & $\begin{array}{c}3,08 * * * \\
(-31,5)\end{array}$ & $\begin{array}{c}1,84 * * * \\
(-5,53)\end{array}$ & $\begin{array}{c}2,17 * * * \\
(-6,08)\end{array}$ \\
\hline Lambda & $\begin{array}{c}0,17 * * * \\
(-3,32)\end{array}$ & & $\begin{array}{l}0,22 * \\
(-2,2)\end{array}$ & $\begin{array}{l}0,15^{* *} \\
(-2,68)\end{array}$ & $\begin{array}{c}0,18 * * * \\
(-3,54)\end{array}$ & & $\begin{array}{l}0,27 * * \\
(-2,84)\end{array}$ & $\begin{array}{l}0,15^{* *} \\
(-2,59)\end{array}$ \\
\hline Sigma2 & $\begin{array}{l}0,27 * * * \\
(-10,44)\end{array}$ & $\begin{array}{l}0,28 * * * \\
(-10,42)\end{array}$ & $\begin{array}{l}0,27 * * * \\
(-10,41)\end{array}$ & $\begin{array}{l}0,27 * * * \\
(-10,43)\end{array}$ & $\begin{array}{l}0,26 * * * \\
(-10,44)\end{array}$ & $\begin{array}{l}0,26 * * * \\
(-10,38)\end{array}$ & $\begin{array}{l}0,26 * * * \\
(-10,39)\end{array}$ & $\begin{array}{l}0,26 * * * \\
(-10,42)\end{array}$ \\
\hline Rho & & $\begin{array}{c}0,23^{*} \\
(-2,35)\end{array}$ & & $\begin{array}{c}0.11 \\
(-0,91)\end{array}$ & & $\begin{array}{l}0,28 * * \\
(-2,99)\end{array}$ & & $\begin{array}{c}0.16 \\
(-1,45)\end{array}$ \\
\hline Observaciones & 218 & 218 & 218 & 218 & 218 & 218 & 218 & 218 \\
\hline
\end{tabular}

Estadístico t entre paréntesis. * Probabilidad $<0.05,{ }^{* *}$ probabilidad $<0.01,{ }^{* * *}$ probabilidad $<0.001$

En el modelo SAR, el coeficiente $\lambda$ es positivo y estadísticamente significativo, lo cual indica que la tasa de emprendimiento de un cantón depende positivamente de la de los cantones vecinos, en concordancia con el trabajo realizado por Yolac (2015). Una posible explicación implica que, cuando en un cantón nace una empresa concentrada en generar determinados productos o servicios, demanda insumos de las zonas aledañas, lo que conllevará a la dinamización económica de forma conjunta. El método SEM, al obtener el coeficiente $\rho$ positivo y significativo, señala que el emprendimiento de un cantón se subordina a las variables omitidas sus vecinos, de ahí la necesidad de incluir variables adicionales en las estimaciones. En el modelo SDM, dado que $\lambda$ es positiva y estadísticamente significativa, se muestra que el emprendimiento de un cantón depende del capital humano de las zonas contiguas, tal como lo mencionan Batjargal (2007) y Lin y Yang (2017). Finalmente, el método SARMA arroja un coeficiente $\lambda$ positivo y significativo, sin embargo, el coeficiente $\rho$ resulta estadísticamente no significativo.

La Tabla 5 muestra la relación entre la tasa de emprendimiento de cinco y diez años en función del capital humano y las variables de control, el conjunto de covariantes. El capital humano mantiene el efecto positivo y estadísticamente significativo, pero disminuye brevemente el tamaño, como era esperado. Los coeficientes de las variables de control son positivos y estadísticamente significativos para SAR y SEM. El primer resultado concuerda con el obtenido en el estudio 
realizado por Lucas y Fuller (2017): revela que la tasa de emprendimiento de una región depende de la tasa de las zonas vecinas; evidentemente, las dotaciones de la fuerza laboral incrementan la productividad $\mathrm{y}$, en general, contribuyen al crecimiento de las empresas (Onkelinx, Manolova y Edelman, 2016). La inclusión de las variables de control en el modelo SDM elimina la significancia estadística, lo que indica que el emprendimiento no siempre dependerá del capital humano de los cantones vecinos, como mencionan Yang y An (2002) y BackesGellner y Moog (2013); no obstante, son estadísticamente significativas al 10\%; esto implica que las variables de control tienen capacidad explicativa de la tasa de emprendimiento regional. Cuando la variable de nacimiento de empresas es de 10 años, los resultados prácticamente se mantienen idénticos.

Tabla 5

RESULTADOS DE LOS MODELOS ESPACIALES CON VARIABLES DE CONTROL

\begin{tabular}{|c|c|c|c|c|c|c|c|c|}
\hline & SAR5 & SEM5 & SDM5 & SARMA5 & SAR10 & SEM10 & SDM10 & SARMA10 \\
\hline $\begin{array}{l}\text { Capital } \\
\text { humano }\end{array}$ & $\begin{array}{c}0,572 * * * \\
(-19,92)\end{array}$ & $\begin{array}{c}0,563 * * * \\
(-18,81)\end{array}$ & $\begin{array}{c}0,572 * * * \\
(-19,96)\end{array}$ & $\begin{array}{c}0,569 * * * \\
(-19,51)\end{array}$ & $\begin{array}{c}0,571^{* * *} \\
-20.44\end{array}$ & $\begin{array}{c}0,561 * * * \\
-19.44\end{array}$ & $\begin{array}{c}0,572 * * * \\
-20.57\end{array}$ & $\begin{array}{c}0,567^{* * *} \\
-20\end{array}$ \\
\hline Exportaciones & $\begin{array}{l}0,10 * * \\
(-2,83)\end{array}$ & $\begin{array}{l}0,12 * * \\
(-3,14)\end{array}$ & $\begin{array}{l}0,11 * * \\
(-2,86)\end{array}$ & $\begin{array}{c}0,11^{* *} \\
(-2,9)\end{array}$ & $\begin{array}{c}0,113^{* *} \\
-3.17\end{array}$ & $\begin{array}{c}0,125 * * * \\
-3.54\end{array}$ & $\begin{array}{c}0,116 * * \\
-3.26\end{array}$ & $\begin{array}{c}0,117^{* * *} \\
-3.3\end{array}$ \\
\hline Ingreso p.c. & $\begin{array}{c}0,159 * * \\
(-3,08)\end{array}$ & $\begin{array}{c}0,142^{* *} \\
(-2,92)\end{array}$ & $\begin{array}{c}0,147^{* *} \\
(-3,05)\end{array}$ & $\begin{array}{c}0,151^{* *} \\
(-3,12)\end{array}$ & $\begin{array}{c}0,150 * * \\
-3.2\end{array}$ & $\begin{array}{c}0,145^{* *} \\
-3.1\end{array}$ & $\begin{array}{c}0,147^{* *} \\
-3.14\end{array}$ & $\begin{array}{c}0,153^{* *} \\
-3.27\end{array}$ \\
\hline Capac. y Form. & $\begin{array}{c}-0,077^{* * *} \\
(-5,27)\end{array}$ & $\begin{array}{c}-0,078^{* * *} \\
(-5,17)\end{array}$ & $\begin{array}{c}-0,076 * * * \\
(-5,24)\end{array}$ & $\begin{array}{c}-0,077 * * * \\
(-5,22)\end{array}$ & $\begin{array}{c}-0,075 * * * \\
(-5,30)\end{array}$ & $\begin{array}{c}-0,076^{* * *} \\
(-5,18)\end{array}$ & $\begin{array}{c}-0,074^{* * *} \\
(-5,25)\end{array}$ & $\begin{array}{c}-0,0752 * * * \\
(-5,23)\end{array}$ \\
\hline Urbanización & $\begin{array}{c}0,67^{* * *} \\
(-3,44)\end{array}$ & $\begin{array}{c}0,79 * * * \\
(-3,98)\end{array}$ & $\begin{array}{c}0,67 * * * \\
(-3,44)\end{array}$ & $\begin{array}{c}0,71 * * * \\
(-3,54)\end{array}$ & $\begin{array}{c}0,66 * * * \\
-3.47\end{array}$ & $\begin{array}{c}0,78^{* * *} \\
-4.02\end{array}$ & $\begin{array}{c}0,66 * * * \\
-3.49\end{array}$ & $\begin{array}{c}0,71^{* * *} \\
-3.64\end{array}$ \\
\hline Constant & $\begin{array}{c}1,35^{* * *} \\
(-3,37)\end{array}$ & $\begin{array}{c}2,18^{* * *} \\
(-7,78)\end{array}$ & $\begin{array}{l}1,29 * * \\
(-3,15)\end{array}$ & $\begin{array}{c}1,47^{* * *} \\
(-3,44)\end{array}$ & $\begin{array}{c}1,66 * * * \\
-4.17\end{array}$ & $\begin{array}{c}2,58^{* * *} \\
-9.56\end{array}$ & $\begin{array}{c}1,53^{* * *} \\
-3.66\end{array}$ & $\begin{array}{c}1,88^{* * *} \\
-4.27\end{array}$ \\
\hline Lambda & $\begin{array}{l}0,14 * * \\
(-3,04)\end{array}$ & & $\begin{array}{c}0.18 \\
(-1,94)\end{array}$ & $\begin{array}{l}0,12^{*} \\
(-2,19)\end{array}$ & $\begin{array}{c}0,14^{* *} \\
-3.25\end{array}$ & & $\begin{array}{c}0,23 * * \\
-2.64\end{array}$ & $\begin{array}{l}0,11^{*} \\
-2.02\end{array}$ \\
\hline Sigma & $\begin{array}{c}0,197^{* * *} \\
(-10,44)\end{array}$ & $\begin{array}{c}0,198 * * * \\
(-10,39)\end{array}$ & $\begin{array}{c}0,196 * * * \\
(-10,42)\end{array}$ & $\begin{array}{c}0,195 * * * \\
(-10,43)\end{array}$ & $\begin{array}{c}0,19 * * * \\
-10.44\end{array}$ & $\begin{array}{c}0,184 * * * \\
-10.37\end{array}$ & $\begin{array}{c}0,184 * * * \\
-10.41\end{array}$ & $\begin{array}{c}0,183^{* * *} \\
-10.41\end{array}$ \\
\hline Rho & & $\begin{array}{c}0,25 * \\
(-2,57)\end{array}$ & & $\begin{array}{c}0.14 \\
(-1,2)\end{array}$ & & $\begin{array}{c}0,31 * * \\
-3.28\end{array}$ & & $\begin{array}{c}0.2 \\
-1.82\end{array}$ \\
\hline Observaciones & 218 & 218 & 218 & 218 & 218 & 218 & 218 & 218 \\
\hline
\end{tabular}

Estadístico t entre paréntesis. ${ }^{*}$ Probabilidad $<0.05,{ }^{* *}$ probabilidad $<0.01, * * *$ probabilidad $<0.001$

En resumen, los resultados obtenidos mediante los modelos básicos y los modelos con variables de control son consistentes: solo se dan ligeros cambios que llevan a conclusiones similares; el capital humano influye positivamente en la tasa de emprendimiento en los diferentes cantones del país; además, la tasa de emprendimiento de una región depende de la correspondiente a los cantones vecinos. Los coeficientes 
obtenidos de la tasa de urbanización, positivos y estadísticamente significativos, se pueden interpretar como el beneficio de la concentración poblacional en los centros urbanos; esto implica que los emprendedores se benefician de la proximidad de tener a los consumidores localizados en las ciudades. Tales resultados son similares a las conclusiones obtenidas por Estrin, Mikiewicz y Stephan (2016), Lin y Yang (2017) y Silveira-Pérez, Cabeza-Pullés y Fernández-Pérez (2016). Los trabajos realizados por Hsiao, Lee y Chen (2016) y Hsiao, et al. (2013) indican que el capital humano constituye un instrumento clave para la creación de pequeñas y medianas empresas, como el capital social para la movilización de recursos e instauración de emprendimientos exitosos.

Los trabajos de Huggins, Prokop y Thompson (2017) señalan que el capital humano permite la peranencia de las empresas a largo plazo, ya que al existir mano de obra cualificada la productividad tenderá a aumentar con el paso del tiempo. Gimmon y Levie (2009) y Van Leeuwen, Van Leeuwen-Li y Foldvari (2017) argumentan que el capital humano y el físico dan paso a una iniciativa empresarial, al progreso tecnológico y al rápido crecimiento de las regiones de un país. Finalmente, Alvarado, Peñarreta, Armas y Alvarado (2017) señalan que el acceso al financiamiento juega un papel relevante en el proceso de generación de nuevas empresas.

Los resultados de las investigaciones empíricas pueden servir de insumo para el diseño y aplicación de políticas públicas orientadas a promover el progreso de las regiones menos desarrolladas; implementarlas debe tomar en cuenta dos aspectos: el emprendimiento genera contagio espacial y el capital humano se mueve en el espacio. Investigaciones empíricas recientes (Mirzanti, Simatupang y Larso, 2015) sugieren que, para promover el surgimiento de nuevos emprendimientos, resulta necesaria la existencia de diversos programas enfocados a la formación empresarial y de habilidades, además de incentivos para las pequeñas y medianas empresas que contemplen la adquisición de tecnología apropiada mediante préstamos destinados a facilitar la creación de negocios propios; en este sentido, Li, et al. (2016) y Mthanti y Ojah (2017) señalan que la existencia de préstamos a tasas relativamente bajas por parte de entidades financieras anima a que los emprendedores 
creen sus propias empresas. De Lucia, Balena, Melone y Borri (2016) también indican que los individuos requieren un fácil acceso a entidades financieras para la obtención de recursos, así como que el gobierno debe enfocarse en mejorar la educación impartida con la finalidad de contar con capital humano capacitado a largo plazo. Por su parte, Cueva y Alvarado (2017) destacan la importancia de la desconcentración del capital humano para optimizar el comportamiento económico de las regiones y para, en particular, abatir la desigualdad de ingresos. Finalmente, Ahsan y Haque (2017) muestran que un alto nivel de escolaridad facilita la creación de emprendimientos que permiten mejorar la calidad de vida y, por tanto, ayudan al crecimiento y desarrollo económico del país. En el caso de Ecuador, la educación púbica es financiada por el Estado, incluida la superior (la privada de este nivel resulta parcialmente subvencionada por el sector público), lo que facilita la aplicación de una política económica: aumentar el gasto en educación en las regiones menos desarrolladas para favorecer su progreso.

\section{CONCLUSIONES}

El trabajo de investigación aborda el emprendimiento en función del capital humano, en Ecuador, mediante un enfoque regional con métodos espaciales. En los últimos años en nuestro país se ha dado una pequeña disminución de la actividad emprendedora debido a diferentes problemas a la hora de conformar pequeñas o medianas empresas; encontramos que la tasa de emprendimiento de una región depende de la de las zonas vecinas y del capital humano local más el aledaño. Estos resultados confirman la importancia de considerar la interacción entre unidades territoriales de modo que permitan medir el contagio/derrame espacial con varias implicaciones de política económica. Conviene resaltar que el nacimiento de nuevas empresas requiere de programas de acompañamiento para lograr su consolidación en el mercado; futuras investigaciones podrán enfocarse en el rol que desempeña el acceso al financiamiento en la determinación de la tasa de emprendimiento, en tanto ello es un factor susceptible de contribuir en la comprensión de los mecanismos de un país en desarrollo para generar dinamismo económico de largo plazo. 


\section{Biblografía}

Ahsan, H. y Haque, M. E. (2017), “Threshold effects of human capital: Schooling and economic growth", Economics Letters, 156, pp. 48-52.

Alvarado, R.; Peñarreta, M.; Armas, R. y Alvarado, R. (2017), “Access to financing and regional entrepreneurship in Ecuador: an approach using spatial methods", International Journal of Entrepreneurship, 21 (3), pp. 1-9.

Anselin, L. (1988), "Lagrange multiplier test diagnostics for spatial dependence and spatial heterogeneity", Geographical analysis, 20 (1), pp. 1-17.

Aroca, P. (2000), Econometría espacial: una herramienta para el análisis de la economía regional, presentado en el IV Encuentro de la Red de Economía Social PANAMA, septiembre 5.

Attanasio, O.; Meghir, C.; Nix, E. y Salvati, F. (2017), "Human capital growth and poverty: Evidence from Ethiopia and Peru", Review of Economic Dynamics, 25, pp. 234-259.

Audretsch, D. B. y Lehmann, E. E. (2005), "Does the knowledge spillover theory of entrepreneurship hold for regions?”, Research Policy, 34 (8), pp. 1191-1202.

Backes-Gellner, U. y Moog, P. (2013), “The disposition to become an entrepreneur and the jacks-of-all-trades in social and human capital", The Journal of Socio-Economics, 47, pp. 55-72.

Banco Mundial (2017), Doing Business, Washington, D.C., disponible en línea.

Baronio, A.; Vianco, A. y Rabanal, C. (2012), "Una introducción a la econometría espacial: dependencia y heterogeneidad”, documento de trabajo.

Barro, R. J. (1992), "Human capital and economic growth", Proceedings, $\mathrm{Fe}$ deral Reserve Bank of Kansas City, pp. 199-230.

Batjargal, B. (2007), "Internet entrepreneurship: Social capital, human capital, and performance of Internet ventures in China", Research policy, 36 (5), pp. 605-618.

Bobonis, G. J. y Morrow, P. M. (2014), "Labor coercion and the accumulation of human capital", Journal of Development Economics, 108, pp. 32-53.

Brush, C.; Ali, A.; Kelley, D. y Greene, P. (2017), “The influence of human capital factors and context on women's entrepreneurship: Which matters more?", Journal of Business Venturing Insights, 8, pp. 105-113.

Cantú, C. (2017), "Entrepreneurial knowledge spillovers: discovering opportunities through understanding mediated spatial relationships", Industrial Marketing Management, 61, pp. 30-42.

Chasco, M. C. (2003), Econometría espacial aplicada a la predicción-extrapolación de datos microterritoriales. Dirección General de Economía y Planificación. Universidad Autónoma de Madrid. 
Cueva, K. y Alvarado, R. (2017), "Concentración espacial de capital humano calificado y desigualdad regional de ingresos en Ecuador", Paradigma Económico, 9 (1), pp. 5-26.

De Lucia, C.; Balena, P.; Melone, M. R. S. y Borri, D. (2016), "Policy, entrepreneurship, creativity and sustainability: The case of "Principi Attivi'('Active Ingredients') in Apulia Region (southern Italy)", Journal of Cleaner Production, 135, pp. 1461-1473.

Devece, C.; Peris-Ortiz, M. y Rueda-Armengot, C. (2016), "Entrepreneurship during economic crisis: Success factors and paths to failure", Journal of Business Research, 69 (11), pp. 5366-5370.

Díaz-García, M. C. y Jiménez-Moreno, J. (2010), “Entrepreneurial intention: the role of gender", International Entrepreneurship and Management Journal, 6 (3), pp. 261-28.

Dutta, N. y Sobel, R. S. (2018), "Entrepreneurship and human capital: The role of financial development", International Review of Economics and Finance, en prensa.

Estrin, S.; Mickiewicz, T. y Stephan, U. (2016), "Human capital in social and commercial entrepreneurship", Journal of Business Venturing, 31 (4), pp. 449-467.

Faggian, A. y Mccann, P. (2009), "Human capital and regional development", Handbook of Regional Growth and Development Theories (vol. 1), Massachusetts, Edward Elgar Publishing Limited, pp. 133-148.

Formichella, M. (2004), El concepto de emprendimiento y su relación con la educación, el empleo y el desarrollo local, Buenos Aires.

GEM (Global Entrepreneurship Monitor) (2016), Global Report, Global Entrepreneurship Research Association, Londres.

Gimmon, E. y Levie, J. (2009), "Instrumental value theory and the human capital of entrepreneurs", Journal of Economic Issues, 43 (3), pp. 715-732.

Hsiao, C., Lee, Y. H. y Chen, H. H. (2016), "The effects of internal locus of control on entrepreneurship: the mediating mechanisms of social capital and human capital", The International Journal of Human Resource Management, 27 (11), pp. 1158-1172.

Hsiao, Y. C.; Hung, S. C.; Chen, C. J. y Dong, T. P. (2013), “Mobilizing human and social capital under industry contexts to pursue high-tech entrepreneurship", Innovation, 15 (4), pp. 515-532.

Huachizaca, V. y Alvarado, R. (2018), "Especialización, diversificación y localización sectorial en Ecuador y su incidencia en el ingreso regional", Regional and Sectoral Economic Studies, 18 (1), pp. 65-80.

Huggins, R.; Prokop, D. y Thompson, P. (2017), "Entrepreneurship and the determinants of firm survival within regions: human capital, growth motivation and locational conditions", Entrepreneurship y Regional Development, 29 (3-4), pp. 357-389. 
INEC (Instituto Nacional de Estadística y Censos) (2010), Censo de población y vivienda, Quito, disponible en línea.

Jiménez, A.; Palmero-Cámara, C.; González-Santos, M. J.; González-Bernal, J. y Jiménez-Eguizábal, J. A. (2015), “The impact of educational levels on formal and informal entrepreneurship", BRQ Business Research Quarterly, 18 (3), pp. 204-212.

Jiménez, J. y Alvarado, R. (2018), "Effect of labor productivity and human capital on regional poverty in Ecuador", Journal of Regional Research, 40 (1), pp. 141-165.

Jiménez, S. y Alvarado, R. (2017), “Especialización sectorial, capital humano e ingreso regional en Ecuador", Revista de Estudios Regionales, en prensa.

Läpple, D.; Renwick, A.; Cullinan, J. y Thorne, F. (2016), "What drives innovation in the agricultural sector? A spatial analysis of knowledge spillovers", Land Use Policy, 56, pp. 238-250.

Lee, S. I. (2001), "Developing a bivariate spatial association measure: an integration of Pearson's r and Moran's I', Journal of Geographical Systems, 3, pp. 369-385

Li, C.; Shi, Y.; Wu, C.; Wu, Z. y Zheng, L. (2016), "Policies of promoting entrepreneurship and Angel Investment: Evidence from China", Emerging Markets Review, 29, pp. 154-167.

Lim, D. S.; Oh, C. H. y De Clercq, D. (2016), "Engagement in entrepreneurship in emerging economies: Interactive effects of individual-level factors and institutional conditions", International Business Review, 25 (4), pp. 933-945.

Lin, X. y Yang, X. (2017), "From human capital externality to entrepreneurial aspiration: Revisiting the migration-trade linkage", Journal of World Business, 52 (3), pp. 360-371.

Lucas, D. S. y Fuller, C. S. (2017), "Entrepreneurship: Productive, unproductive, and destructive-Relative to what?", Journal of Business Venturing Insights, 7, pp. 45-49.

Lucas, R. (1988), "On the mechanics of economic development", Journal of Monetary Economics, 22, pp. 3-42.

Mankiw, N. G.; Romer, D. y Weil, D. N. (1992), "A contribution to the empirics of economic growth", The quarterly journal of economics, 107 (2), pp. 407-437.

Martin, B. C.; McNally, J. J. y Kay, M. J. (2013), "Examining the formation of human capital in entrepreneurship: A meta-analysis of entrepreneurship education outcomes", Journal of Business Venturing, 28 (2), pp. 211-224.

Marulanda-Valencia, F. Á.; Montoya-Restrepo, I. A. y Vélez-Restrepo, J. M. (2014), "Teorías motivacionales en el estudio del emprendimiento", Pensamiento y Gestión, (36), pp. 204-236. 
Mejía-Cubillos, J. (2013), "Interregional links in Colombian economy during the 19th century: Caribbean entrepreneurship in the inland",

Mestieri, M.; Schauer, J. y Townsend, R. M. (2017), "Human capital acquisition and occupational choice: Implications for economic development", Review of Economic Dynamics, 25, pp. 151-186.

Mirzanti, I. R.; Simatupang, T. M. y Larso, D. (2015), "Mapping on entrepreneurship policy in Indonesia", Procedia-Social and Behavioral Sciences, 169 , pp. 346-353.

Mora, E.; Vicente, I.; Villegas, P. y Alvarado, R. (2017), “Relación entre el capital humano y el producto en Ecuador: el rol de las políticas educativas", Atlantic Review of Economics, 1, pp. 1-19.

Moreno-Serrano, R. y Vayá, E. V. (2002), “Econometría espacial: nuevas técnicas para el análisis regional. Una aplicación a las regiones europeas", Investigaciones regionales, (1), pp. 83-106.

Mthanti, T. y Ojah, K. (2017), "Entrepreneurial orientation (EO): Measurement and policy implications of entrepreneurship at the macroeconomic level", Research Policy, 46 (4), pp. 724-739.

Onkelinx, J.; Manolova, T. S. y Edelman, L. F. (2016), “The human factor: Investments in employee human capital, productivity, and SME internationalization", Journal of International Management, 22 (4), pp. 351-364.

Piacentino, D.; Bono, F.; Cracolici, M. F. y Giuliani, D. (2017), “A spatial analysis of new business formation: Replicative vs innovative behavior", Spatial Statistics, vol. 21, pp. 390-405.

Plummer, L. A. y Acs, Z. J. (2014), "Localized competition in the knowledge spillover theory of entrepreneurship", Journal of Business Venturing, 29 (1), pp. 121-136.

Qian, H. y Zhao, C. (2018), "Space-time analysis of high technology entrepreneurship: A comparison of California and New England", Applied Geography, 95, pp. 111-119.

Salazar, A. L.; Hidalgo, J. F. O. y Manríquez, M. R. (2017), “La responsabilidad social empresarial desde la percepción del capital humano. Estudio de un caso", Revista de Contabilidad, 20 (1), pp. 36-46.

SIISE (2008), Sistema Integrado de Indicadores Sociales del Ecuador, Ministerio de Coordinación de Desarrollo Social. Quito.

Silveira-Pérez, Y.; Cabeza-Pullés, D. y Fernández-Pérez, V. (2016), “Emprendimiento: perspectiva cubana en la creación de empresas familiares", $E u$ ropean Research on Management and Business Economics, 22 (2), pp. 70-77.

Urbano, D. y Aparicio, S. (2016), "Entrepreneurship capital types and economic growth: International evidence", Technological Forecasting and Social Change, 102, pp. 34-44. 
Van Leeuwen, B.; Van Leeuwen-Li, J. y Foldvari, P. (2017), "Human capital in Republican and New China: regional and long-term trends", Economic History of Developing Regions, 32 (1), pp. 1-36.

Weldeegzie, S. G. (2017), "Growing-up unfortunate: War and Human Capital in Ethiopia", World Development, 96, pp. 474-489.

Wu, C. W. y Huarng, K. H. (2015), "Global entrepreneurship and innovation in management", Journal of Business Research, 68 (4), pp. 743-747.

Yang, D. T. y An, M. Y. (2002), "Human capital, entrepreneurship, and farm household earnings", Journal of Development Economics, 68 (1), pp. 6588.

Yolac, S. (2015), “An Empirical Study Regarding Entrepreneurship in Europe and Central Asia", Procedia-Social and Behavioral Sciences, 195, pp. 1097-1103. 